Angulo, A., Martínez-Servín, F., Torres-Hernández, E., Domínguez-Domínguez, O., \& Cortés, J. (2021). Ictiofauna de la zona intermareal rocosa del Área de Conservación Guanacaste, Pacífico Norte de Costa Rica: diversidad y aspectos ecológicos y biogeográficos. Revista de Biología Tropical, 69(Suppl. 2), S277-S296. https://doi. org/10.15517/rbt.v69iS2.48320

https://doi.org/10.15517/rbt.v69iS2.48320

\title{
Ictiofauna de la zona intermareal rocosa del Área de Conservación Guanacaste, Pacífico Norte de Costa Rica: diversidad y aspectos ecológicos y biogeográficos
}

Arturo Angulo ${ }^{1,2}$; (D) https://orcid.org/0000-0002-4587-1446

Francisco Martínez-Servín3; (D) https://orcid.org/0000-0001-7099-5304

Eloísa Torres-Hernández ${ }^{4}$; (D) https://orcid.org/0000-0002-8518-6750

Omar Domínguez-Domínguez ${ }^{3}$; (D) https://orcid.org/0000-0001-9583-4443

Jorge Cortés ${ }^{1,2}$; (D) https://orcid.org/0000-0001-7004-8649

1. Museo de Zoología, Centro de Investigación en Biodiversidad y Ecología Tropical (CIBET), Universidad de Costa Rica, 11501-2060, San José, Costa Rica; arturo.angs@gmail.com

2. Centro de Investigación en Ciencias del Mar y Limnología (CIMAR), Universidad de Costa Rica, 11501-2060, San José, Costa Rica; jorge.cortes@ucr.ac.cr

3. Laboratorio de Biología Acuática, Facultad de Biología, Universidad Michoacana de San Nicolás de Hidalgo. Edificio "R" planta baja, Ciudad Universitaria, 58030 Morelia, Michoacán, México; franciscomartinezservin@gmail.com; godeido@yahoo.com.mx

4. Posgrado en Ciencias del Mar y Limnología, Universidad Nacional Autónoma de México. Avenida Ciudad Universitaria 3000, C. P. 04510, Coyoacán, Ciudad de México, México; eltorres@gmail.com

Recibido 25-I-2021. Revisado 14-II-2021. Aceptado 12-V-2021.

\author{
ABSTRACT \\ Ichthyofauna of the rocky intertidal zone of the Guanacaste Conservation Area, \\ North Pacific of Costa Rica; diversity and ecological and biogeographic aspects
}

\begin{abstract}
Introduction: The rocky intertidal constitutes a dominant coastal seascape in the Eastern Tropical Pacific, harboring highly diverse and functionally complex biological communities. The knowledge about the diversity and the diverse biological processes that occur in such environments is, however, quite limited on a regional scale. Objective: Quantify, describe and compare (on a regional scale) the ichthyological diversity associated with the rocky intertidal zone of the Guanacaste Conservation Area (ACG), Costa Rica, as well as to evaluate and discuss some biogeographic and ecological patterns.
\end{abstract}

Methods: A total of 49 tide pools were sampled between 2014 and 2019, at seven locations within the ACG. Data on absolute diversity (presence/absence of species) were obtained, in addition to some ecological data (residence status, development stage (s), trophic guild, aggregation and cryptic habits and generalized geographic distribution), these data were compared with published records from other surveys/inventories carried out at various locations (20) along the Eastern Pacific coast. Through a similarity analysis, both possible clustering patterns between localities (according to their species composition) and previous hypotheses of regionalization, under a biogeographic perspective, were evaluated.

Results: A total of 67 species of bony fish were recorded in the rocky intertidal area of the ACG, being this the most diverse locality (with published data) in the Eastern Pacific. The components/categories "transitory" with 30 species (44.8\%), "juveniles" with 38 species (55.9\%), "carnivores" with 47 species (70.1\%), "solitary" with 32 species (47.8\%) and "endemic to the ETP" with 66 species (98\%), were dominant (in terms of the total number of species) within the ACG. The results of the similarity analysis supported the biogeographic division 
scheme, defined a priori; with the localities within the Californian, Cortez, Mexican, Panamic and PeruvianChilean provinces recovered as highly consistent groups. This structure is consistent with the effect of barriers such as the sandy gaps of Sinaloa and Central America, among others, limiting the flow of species between biogeographic units.

Conclusions: The ACG has a high ichthyological diversity associated with its rocky intertidal zone, which is product of a unique combination of factors at the regional level. The results of this study reinforce the need to carry out more basic and applied studies with the aim of helping to better understand and know different key aspects of the dynamics and physical-chemical-biological processes occurring in intertidal zones, of its associated biological diversity, of its importance within the marine-coastal landscape as a continuum and of the possible effects that these environments could suffer due to the impact of human activities.

Key words: biogeography; Eastern Tropical Pacific; Central America; ichthyofauna; fishes; tide pools.

El intermareal rocoso constituye un paisaje marino costero dominante, en términos del área total ocupada, en múltiples regiones del globo, albergando comunidades biológicas altamente diversas y funcionalmente complejas (Castellanos-Galindo, Giraldo, \& Zapata, 2014; Gibson \& Yoshiyama, 1999; Horn \& Gibson, 1988). Los diversos ambientes/ecosistemas ubicados dentro de este tipo de paisaje son a su vez caracterizados, definidos y afectados por una serie de condiciones, variables y presiones, del tipo biológicas, físicas y químicas, tanto exógenas como endógenas, en extremo fluctuantes; esto como resultado, en primera instancia, de los cambios diarios y estacionales asociados con el régimen de mareas y de radiación solar, entre otros (Almada \& Faria, 2004; Arakaki \& Tokeshi, 2006; Castellanos-Galindo et al., 2014; Gibson \& Yoshhiyama, 1999). Las especies ícticas habitando tales ambientes/ ecosistemas presentan, en consecuencia, una gran variedad de rasgos morfológicos externos e internos, conductuales y/o fisiológicos, como adaptaciones puntuales y en muchos casos únicas a este conjunto de condiciones y presiones, permitiendo así su supervivencia (Castellanos-Galindo, Giraldo, \& Rubio, 2005; Gibson, 1982; Horn \& Gibson, 1988; Quijada \& Caceres, 2000). Esto aplica, en mayor o menor medida a especies residentes, pero también a aquellas denominadas "transitorias" o "visitantes accidentales" sensu Gibson (1982).

La región biogeográfica marina conocida como el Pacífico Tropical Oriental (PTO), la cual incluye parte importante de la costa oeste del continente americano (i.e., desde el sur de Baja California y la porción norte del Golfo de
California, México, hasta el norte de Perú), así como diversas islas oceánicas, archipiélagos $\mathrm{y}$ atolones (Revillagigedo, Clipperton, Coco, Malpelo y Galápagos), presenta una elevada diversidad biológica. En lo que respecta al componente ictiológico, se han reportado alrededor de 1360 especies (esto solo entre los $0 \mathrm{y}$ $100 \mathrm{~m}$ de profundidad), de las cuales alrededor de un $44 \%$ ocupan, temporal o permanentemente, ambientes rocosos costeros (Robertson \& Allen, 2015). El intermareal rocoso, de hecho, cubre entre un 15 y un $20 \%$ de la línea de costa del PTO, siendo el paisaje marino costero dominante en áreas tales como la porción suroeste de Baja California Sur, la porción central de México, el norte de Costa Rica y el norte de Colombia, entre otros; así como en la totalidad de las islas oceánicas (Castellanos-Galindo et al., 2005; Castellanos-Galindo et al., 2014; Robertson \& Allen, 2015; Weaver, 1970).

Pese a la importancia del intermareal rocoso en términos de la diversidad biológica, morfológica y funcional que alberga, así como a la complejidad y valor (ecológico y económico) de los procesos que en él se desarrollan (e.g., como zona de crianza, reproducción y alimentación de especies de interés comercial, entre otros), este es un ambiente relativamente poco estudiado; y, en última instancia, el PTO no es la excepción (Castellanos-Galindo et al., 2005; Castellanos-Galindo, 2014; GonzálezMurcia, Chicas-Batres, \& Lovo, 2016; González-Murcia, Marín-Martínez, \& Ayala-Bocos, 2012; Weaver, 1970). En concordancia, son relativamente escasos los estudios básicos y aplicados, considerando aspectos ecológicos, biogeográficos y más aún de inventariado (i.e., 
taxonómicos), a lo largo de la costa rocosa del PTO. Cabe destacar, sin embargo, los aportes, en orden cronológico, de autores tales como Weaver (1970); Thomson y Lehner (1976); Almada y Faria (2004); Castellanos-Galindo et al. (2005); Castellanos-Galindo \& Giraldo (2008); Ruiz-Campos et al., (2010); GonzálezMurcia et al. (2012); Castellanos-Galindo et al. (2014); González-Murcia et al. (2016); BarjauGonzález, Romo-Piñeda, López-Vivas, PérezCastillo, y Milicua (2017); Barjau-González, Armenta-Quintana, López-Vivas y RomeroVadillo (2019); y González-Murcia, Erdman, y Larios (2020), entre otros, quienes han contribuido, de una u otra forma, a mejorar nuestro entendimiento sobre diversos aspectos (e.g., composición específica, ecología trófica, biogeografía, entre otros) de la fauna íctica asociada a este tipo de ambientes, a diferentes escalas locales e incluso con aproximaciones y análisis comparativos a nivel regional.

El presente estudio, en correspondencia con lo anterior, tiene por objetivo general cuantificar y describir la diversidad ictiológica asociada al intermareal rocoso del Área de Conservación Guanacaste (ACG), Pacífico norte de Costa Rica - un área protegida estatal, la cual, en años recientes, ha redoblado esfuerzos para inventariar y caracterizar su flora y fauna marina (Cortés, 2017; Cortés \& Joyce, 2020) - a efectos de contribuir a un mejor entendimiento de la diversidad local y regional. En esta contribución realizamos además un ejercicio de comparación, tomando como base de referencia datos de riqueza absoluta y distribución de especies recopilados a partir de diversos estudios publicados (y sin publicar), incluyendo trabajos de inventario básico y/o aplicados, llevados a cabo en diversas localidades dentro de los límites del PTO (y fuera de estos, pero siempre dentro de la región Pacífico Oriental), discutiendo algunas implicaciones y aspectos ecológicos y biogeográficos. Entre los objetivos específicos planteados, dado lo anterior, se listan: 1) determinar áreas con la mayor riqueza de especies intermareales en el PTO, y 2) evaluar, desde un contexto ecológico y biogeográfico, las posibles diferencias o similitudes entre los ensambles de peces intermareales (en términos de su riqueza de especies) en diversas localidades dentro de los límites del PTO.

\section{MATERIALES Y MÉTODOS}

Inventariado: Los muestreos se realizaron entre 2014 y 2019 en un total de siete puntos o ubicaciones [Playa La Isla, Junquillal (JU; $10^{\circ} 57^{\prime} 49.92$ " N \& 8541'40.74” O), Bahía Cuajiniquil (CU; $10^{\circ} 56^{\prime} 4.26^{\prime \prime} \mathrm{N} \& 85^{\circ} 42^{\prime} 8.57^{\prime \prime}$ O), Bahía Santa Elena (SE; 1054’38.16” N \& $85^{\circ} 47^{\prime} 47.27^{\prime \prime}$ O), Bahía Santa Elena, Sortija (SO; 1055'50.78” N \& 8549'5.18” O), Isla San José (SJ; 1051'26.92” N \& 8554'43.88” O), Bahía Potrero Grande (PG; 1050’46.17” N \& 8547'17.92” O) y Playa Naranjo (PN; $10^{\circ} 47^{\prime} 19.86^{\prime \prime} \mathrm{N} \& 85^{\circ} 40^{\prime} 26.51$ ' O)] distribuidos a lo largo de la línea de costa del ACG (Fig. 1, Tabla 1). Estos siete puntos se visitaron al menos dos veces en este periodo de cinco años, durante los meses de febrero-marzo (estación seca) y julio-agosto (estación lluviosa), respectivamente. En los diferentes sitios se seleccionaron arbitrariamente, pero cubriendo todo el eje vertical desde la bajamar hasta la pleamar, así como la mayor diversidad de microambientes posibles, un total de 49 pozas de marea (JU: 11, CU: 13, SE: 9, SO: 5, SJ: 4, PG: 3, PN: 4), muestreadas una única vez por estación. Las pozas se muestrearon con el uso de redes de mano y una solución 2: 8 de aceite de clavo (euglenol): etanol $96 \%$ como anestésico (Castellanos-Galindo et al., 2014; Robertson \& Smith-Vaniz, 2008). Esta solución fue adicionada, en una proporción razonable, según el volumen total de agua de cada poza, cubriendo la totalidad de su área superficial. Esto a efectos de facilitar la captura de los ejemplares ictiológicos, así como para maximizar la representatividad de los muestreos; dada la posible presencia de organismos/especies con patrones de coloración y hábitos crípticos usualmente no detectables por otros medios/técnicas de muestreo (e.g., censos visuales o captura con redes).

Ejemplares de referencia (previamente anestesiados) fueron trasladados a un 


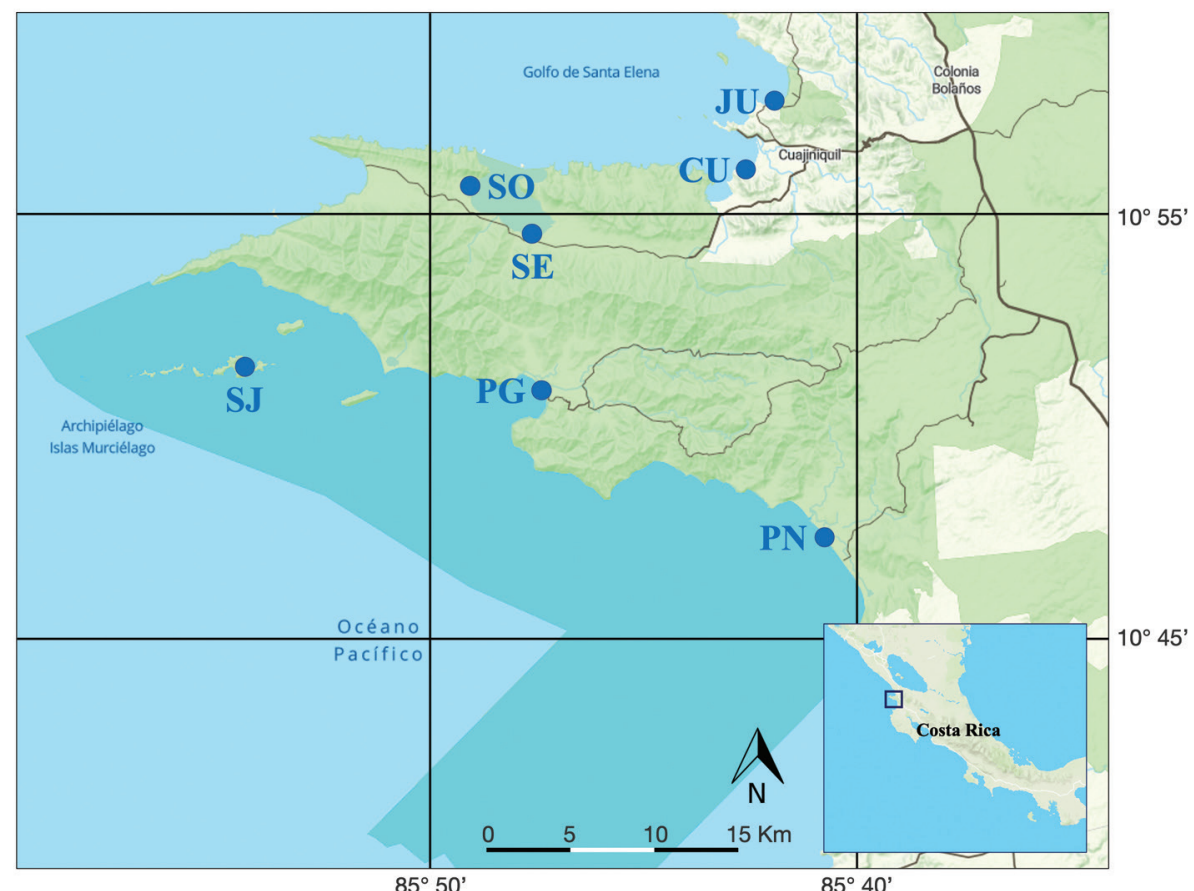

Fig. 1. Puntos de muestreo a lo largo de la línea de costa del ACG, Pacífico Norte de Costa Rica. Playa La Isla, Junquillal (JU), Bahía Cuajiniquil (CU), Bahía Santa Elena (SE), Bahía Santa Elena, Sortija (SO), Isla San José (SJ), Bahía Potrero Grande (PG) y Playa Naranjo (PN).

Fig. 1. Sampling points along the coastline of the ACG, North Pacific coast of Costa Rica. La Isla Beach, Junquillal (JU), Cuajiniquil Bay (CU), Santa Elena Bay (SE), Santa Elena Bay, Sortija (SO), San José Island (SJ), Potrero Grande Bay (PG) and Naranjo Beach $(\mathrm{PN})$.

TABLA 1

Lista de ordenes, familias y especies ícticas asociadas al intermareal rocoso del ACG, según localidad (ver Materiales y Métodos), y datos ecológicos. Abu.: Abundancias relativas según intervalos de capturas (ver detalles en Materiales y Métodos); Est.: Estadio de desarrollo (Juvenil=J o Adulto=A); Gre.: Gremio trófico (Omnívoro=Omn, Carnívoro=Car, Herbívoro=Her, Detritívoro=Det o Planctívoro=Pla); y Com.: Comportamiento $($ Solitario $=\mathrm{S}$, Gregario $=\mathrm{G}$ o Críptico $=\mathrm{C}$ )

TABLE 1

List of fish orders, families and species of fishes associated with the rocky intertidal area of the ACG, according to locality (see Materials and Methods), and ecological data. Abu.: Relative abundances according to catch intervals (see details in Materiales y Métodos); Est.: Development stage (Juvenile=J or Adult=A); Gre.: Trophic guild (Omnivorous $=$ Omn, Carnivorous $=$ Car, Herbivorous $=$ Her, Detritivorous $=$ Det or Planktivorous $=\mathrm{Pla})$; and Com.: Behaviour (Solitarious $=\mathrm{S}$, Gregarious $=\mathrm{G}$ or Criptic $=\mathrm{C}$ )

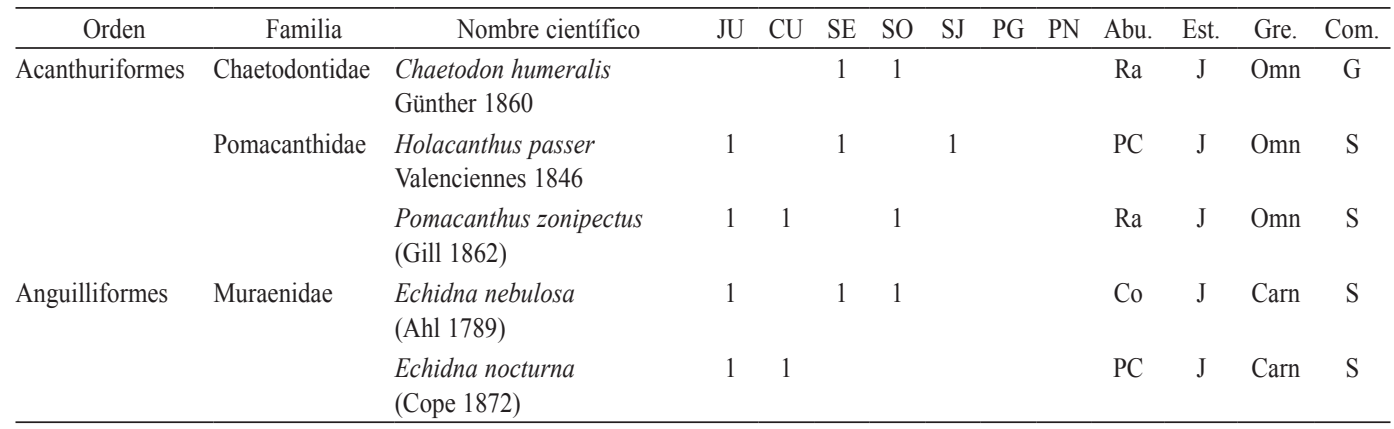




\begin{tabular}{|c|c|c|c|c|c|c|c|c|c|c|c|c|c|}
\hline Orden & Familia & Nombre científico & JU & $\mathrm{CU}$ & SE & SO & SJ & PG & $\mathrm{PN}$ & Abu. & Est. & Gre. & Com. \\
\hline & & $\begin{array}{l}\text { Gymnothorax castaneus } \\
\text { (Jordan \& Gilbert 1883) }\end{array}$ & & & 1 & 1 & & & & $\mathrm{Ra}$ & $\mathrm{J}$ & Carn & $\mathrm{S}$ \\
\hline & & $\begin{array}{l}\text { Gymnothorax dovii } \\
\text { (Günther 1870) }\end{array}$ & 1 & & & & & & & $\mathrm{Ra}$ & $\mathrm{J}$ & Carn & $\mathrm{S}$ \\
\hline \multirow[t]{9}{*}{ Blenniiformes } & Blenniidae & $\begin{array}{l}\text { Ophioblennius steindachneri } \\
\text { Jordan \& Evermann } 1898\end{array}$ & 1 & 1 & 1 & 1 & 1 & & 1 & $\mathrm{MC}$ & $\mathrm{J} / \mathrm{A}$ & $\begin{array}{l}\text { Det/ } \\
\text { Her }\end{array}$ & $\mathrm{S} / \mathrm{G} / \mathrm{C}$ \\
\hline & Chaenopsidae & $\begin{array}{l}\text { Acanthemblemaria } \\
\text { exilispinus } \\
\text { Stephens } 1963\end{array}$ & & 1 & 1 & & & & & $\mathrm{PC}$ & $\mathrm{J} / \mathrm{A}$ & Carn & $\mathrm{S} / \mathrm{C}$ \\
\hline & Labrisomidae & $\begin{array}{l}\text { Dialommus macrocephalus } \\
\text { (Günther 1861) }\end{array}$ & 1 & 1 & 1 & & 1 & & 1 & $\mathrm{MC}$ & $\mathrm{J} / \mathrm{A}$ & Carn & $\mathrm{S} / \mathrm{C}$ \\
\hline & & $\begin{array}{l}\text { Malacoctenus ebisui } \\
\text { Springer } 1959\end{array}$ & 1 & 1 & 1 & 1 & 1 & & & $\mathrm{MC}$ & $\mathrm{J} / \mathrm{A}$ & Carn & $\mathrm{S} / \mathrm{G} / \mathrm{C}$ \\
\hline & & $\begin{array}{l}\text { Malacoctenus margaritae } \\
\text { Fowler 1944) }\end{array}$ & & & 1 & 1 & 1 & & & Co & $\mathrm{J} / \mathrm{A}$ & Carn & $\mathrm{S} / \mathrm{G} / \mathrm{C}$ \\
\hline & & $\begin{array}{l}\text { Malacoctenus sudensis } \\
\text { Springer } 1959\end{array}$ & 1 & 1 & 1 & 1 & 1 & & 1 & $\mathrm{Ab}$ & $\mathrm{J} / \mathrm{A}$ & Carn & $\mathrm{S} / \mathrm{G} / \mathrm{C}$ \\
\hline & & $\begin{array}{l}\text { Starksia fulva } \\
\text { Rosenblatt \& Taylor } 1971\end{array}$ & & & & 1 & 1 & & & $\mathrm{PC}$ & $\mathrm{J} / \mathrm{A}$ & Carn & $\mathrm{S} / \mathrm{C}$ \\
\hline & Tripterygiidae & $\begin{array}{l}\text { Axoclinus lucillae } \\
\text { Fowler } 1944\end{array}$ & & & & & 1 & & & $\mathrm{Ra}$ & $\mathrm{J} / \mathrm{A}$ & Carn & $\mathrm{S} / \mathrm{C}$ \\
\hline & & $\begin{array}{l}\text { Lepidonectes clarkhubbsi } \\
\text { Bussing } 1991\end{array}$ & & & & 1 & 1 & & & $\mathrm{PC}$ & $\mathrm{J} / \mathrm{A}$ & Carn & $\mathrm{S} / \mathrm{C}$ \\
\hline \multirow[t]{3}{*}{ Carangiformes } & Carangidae & $\begin{array}{l}\text { Oligoplites refulgens } \\
\text { Gilbert \& Starks } 1904\end{array}$ & & & & & & 1 & & $\mathrm{Ra}$ & $\mathrm{J}$ & Carn & S \\
\hline & Paralichthyidae & $\begin{array}{l}\text { Citharichthys gilberti } \\
\text { Jenkins \& Evermann } 1889\end{array}$ & & & & & & 1 & & $\mathrm{Ra}$ & $\mathrm{J}$ & Carn & S \\
\hline & & $\begin{array}{l}\text { Cyclopsetta panamensis } \\
\text { (Steindachner 1876) }\end{array}$ & & & & & & 1 & & $\mathrm{Ra}$ & $\mathrm{J}$ & Carn & S \\
\hline Clupeiformes & Clupeidae & $\begin{array}{l}\text { Lile stolifera } \\
\text { (Jordan \& Gilbert 1882) }\end{array}$ & & & & & & 1 & & $\mathrm{Ra}$ & $\mathrm{J} / \mathrm{A}$ & Pla & G \\
\hline \multirow[t]{5}{*}{ Gobiesociformes } & Gobiesocidae & $\begin{array}{l}\text { Arcos decoris } \\
\text { Briggs } 1969\end{array}$ & & 1 & 1 & 1 & 1 & & & Co & $\mathrm{J} / \mathrm{A}$ & Carn & $\mathrm{S} / \mathrm{C}$ \\
\hline & & $\begin{array}{l}\text { Arcos rhodospilus } \\
\text { (Günther 1864) }\end{array}$ & & 1 & & 1 & 1 & & 1 & $\mathrm{MC}$ & $\mathrm{J} / \mathrm{A}$ & Carn & $\mathrm{S} / \mathrm{C}$ \\
\hline & & $\begin{array}{l}\text { Gobiesox adustus } \\
\text { Jordan \& Gilbert } 1882\end{array}$ & 1 & 1 & 1 & 1 & 1 & & 1 & $\mathrm{MC}$ & $\mathrm{J} / \mathrm{A}$ & Carn & $\mathrm{S} / \mathrm{G} / \mathrm{C}$ \\
\hline & & $\begin{array}{l}\text { Gobiesox daedaleus } \\
\text { Briggs } 1951\end{array}$ & 1 & 1 & & & 1 & & 1 & $\mathrm{MC}$ & $\mathrm{J} / \mathrm{A}$ & Carn & $\mathrm{S} / \mathrm{G} / \mathrm{C}$ \\
\hline & & $\begin{array}{l}\text { Tomicodon petersii } \\
\text { (Garman 1875) }\end{array}$ & 1 & 1 & 1 & 1 & 1 & & 1 & $\mathrm{Ab}$ & $\mathrm{J} / \mathrm{A}$ & Carn & $\mathrm{S} / \mathrm{G} / \mathrm{C}$ \\
\hline \multirow[t]{5}{*}{ Gobiiformes } & Gobiidae & $\begin{array}{l}\text { Bathygobius andrei } \\
\text { (Sauvage 1880) }\end{array}$ & & & 1 & & 1 & & 1 & $\mathrm{MC}$ & $\mathrm{J} / \mathrm{A}$ & Carn & $\mathrm{S} / \mathrm{G} / \mathrm{C}$ \\
\hline & & $\begin{array}{l}\text { Bathygobius ramosus } \\
\text { Ginsburg } 1947\end{array}$ & 1 & 1 & 1 & 1 & 1 & 1 & 1 & $\mathrm{Ab}$ & $\mathrm{J} / \mathrm{A}$ & Carn & $\mathrm{S} / \mathrm{G} / \mathrm{C}$ \\
\hline & & $\begin{array}{l}\text { Coryphopterus urospilus } \\
\text { Ginsburg } 1938\end{array}$ & & 1 & & 1 & 1 & & 1 & $\mathrm{PC}$ & $\mathrm{J} / \mathrm{A}$ & Carn & $\mathrm{S} / \mathrm{C}$ \\
\hline & & $\begin{array}{l}\text { Ctenogobius sagittula } \\
\text { (Günther 1862) }\end{array}$ & & & & & & 1 & 1 & $\mathrm{Ra}$ & $\mathrm{J} / \mathrm{A}$ & Her & S \\
\hline & & $\begin{array}{l}\text { Gobionellus microdon } \\
\text { (Gilbert 1892) }\end{array}$ & 1 & 1 & 1 & & & & & $\mathrm{PC}$ & $\mathrm{J} / \mathrm{A}$ & Omn & $\mathrm{S} / \mathrm{C}$ \\
\hline
\end{tabular}




\begin{tabular}{|c|c|c|c|c|c|c|c|c|c|c|c|c|c|}
\hline Orden & Familia & Nombre científico & $\mathrm{JU}$ & $\mathrm{CU}$ & SE & SO & SJ & PG & PN & Abu. & Est. & Gre. & Com. \\
\hline & & $\begin{array}{l}\text { Gobulus crescentalis } \\
\text { (Gilbert 1892) }\end{array}$ & & 1 & & 1 & & 1 & & PC & $\mathrm{J} / \mathrm{A}$ & Carn & $\mathrm{S} / \mathrm{C}$ \\
\hline & & $\begin{array}{l}\text { Gobulus hancocki } \\
\text { Ginsburg } 1938\end{array}$ & & & & & 1 & & & $\mathrm{Ra}$ & $\mathrm{J} / \mathrm{A}$ & Carn & $\mathrm{S} / \mathrm{C}$ \\
\hline & & $\begin{array}{l}\text { Tigrigobius digueti } \\
\text { (Pellegrin 1901) }\end{array}$ & 1 & & 1 & & & & & $\mathrm{PC}$ & $\mathrm{J} / \mathrm{A}$ & Carn & $\mathrm{S} / \mathrm{C}$ \\
\hline & & $\begin{array}{l}\text { Tigrigobius inornatus } \\
\text { Bussing } 1990\end{array}$ & 1 & & 1 & 1 & 1 & & & $\mathrm{PC}$ & $\mathrm{J} / \mathrm{A}$ & Carn & $\mathrm{S} / \mathrm{C}$ \\
\hline & & $\begin{array}{l}\text { Tigrigobius puncticulatus } \\
\text { (Ginsburg 1938) }\end{array}$ & & 1 & & & & & & $\mathrm{PC}$ & $\mathrm{J} / \mathrm{A}$ & Carn & $\mathrm{S} / \mathrm{C}$ \\
\hline Holocentriformes & Holocentridae & $\begin{array}{l}\text { Sargocentron suborbitale } \\
\text { (Gill 1863) }\end{array}$ & 1 & 1 & 1 & 1 & 1 & & 1 & $\mathrm{MC}$ & $\mathrm{J} / \mathrm{A}$ & Carn & $\mathrm{S} / \mathrm{G}$ \\
\hline \multirow[t]{2}{*}{ Mugiliformes } & Mugilidae & $\begin{array}{l}\text { Chaenomugil proboscideus } \\
\text { (Günther 1861) }\end{array}$ & 1 & 1 & & & & 1 & 1 & $\mathrm{MC}$ & $\mathrm{J}$ & $\begin{array}{l}\text { Det/ } \\
\text { Her }\end{array}$ & $\mathrm{S} / \mathrm{G}$ \\
\hline & & Mugil setosus Gilbert 1892 & 1 & 1 & & 1 & & 1 & & $\mathrm{Ab}$ & $\mathrm{J}$ & $\begin{array}{l}\text { Det/ } \\
\text { Her }\end{array}$ & $\mathrm{S} / \mathrm{G}$ \\
\hline \multirow[t]{18}{*}{ Perciformes } & Apogonidae & Apogon dovii Günther 1862 & 1 & 1 & 1 & & & & 1 & $\mathrm{PC}$ & $\mathrm{J} / \mathrm{A}$ & Pla & $\mathrm{S} / \mathrm{C}$ \\
\hline & Gerreidae & $\begin{array}{l}\text { Eucinostomus currani } \\
\text { Zahuranec } 1980\end{array}$ & & & & & & 1 & & $\mathrm{PC}$ & $\mathrm{J}$ & Carn & G \\
\hline & Haemulidae & $\begin{array}{l}\text { Conodon serrifer } \\
\text { Jordan \& Gilbert } 1882\end{array}$ & & & & & 1 & & & $\mathrm{Ra}$ & $\mathrm{J}$ & Carn & $\mathrm{S}$ \\
\hline & & $\begin{array}{l}\text { Haemulon steindachneri } \\
\text { (Jordan \& Gilbert 1882) }\end{array}$ & 1 & & & & & & & $\mathrm{Ra}$ & $\mathrm{J}$ & Carn & $\mathrm{S}$ \\
\hline & Labridae & $\begin{array}{l}\text { Halichoeres chierchiae } \\
\text { Di Caporiacco } 1948\end{array}$ & 1 & 1 & 1 & 1 & 1 & & 1 & Co & $\mathrm{J}$ & Carn & $\mathrm{S}$ \\
\hline & & $\begin{array}{l}\text { Halichoeres dispilus } \\
\text { (Günther 1864) }\end{array}$ & 1 & & 1 & & 1 & & 1 & Co & $\mathrm{J}$ & Carn & S \\
\hline & & $\begin{array}{l}\text { Halichoeres nicholsi } \\
\text { (Jordan \& Gilbert 1882) }\end{array}$ & & & 1 & 1 & & & & Co & $\mathrm{J}$ & Carn & $\mathrm{S}$ \\
\hline & & $\begin{array}{l}\text { Halichoeres notospilus } \\
\text { (Günther 1864) }\end{array}$ & 1 & 1 & 1 & 1 & 1 & & 1 & Co & $\mathrm{J}$ & Carn & $\mathrm{S}$ \\
\hline & & $\begin{array}{l}\text { Thalassoma lucasanum } \\
\text { (Gill 1862) }\end{array}$ & 1 & 1 & 1 & 1 & 1 & & 1 & $\mathrm{MC}$ & $\mathrm{J}$ & Carn & $\mathrm{S} / \mathrm{G}$ \\
\hline & Lutjanidae & $\begin{array}{l}\text { Lutjanus guttatus } \\
\text { (Steindachner 1869) }\end{array}$ & & & & 1 & & & & $\mathrm{Ra}$ & $\mathrm{J}$ & Carn & S \\
\hline & Pomacentridae & $\begin{array}{l}\text { Abudefduf concolor } \\
\text { (Gill 1862) }\end{array}$ & 1 & 1 & 1 & 1 & 1 & & 1 & $\mathrm{Ab}$ & $\mathrm{J}$ & Omn & $\mathrm{S} / \mathrm{G}$ \\
\hline & & $\begin{array}{l}\text { Abudefduf declivifrons } \\
\text { (Gill 1862) }\end{array}$ & 1 & 1 & 1 & & & & & Co & $\mathrm{J}$ & Omn & $\mathrm{S}$ \\
\hline & & $\begin{array}{l}\text { Abudefduf troschelii } \\
\text { (Gill 1862) }\end{array}$ & 1 & 1 & 1 & 1 & 1 & & 1 & $\mathrm{Ab}$ & $\mathrm{J}$ & Omn & $\mathrm{S} / \mathrm{G}$ \\
\hline & & $\begin{array}{l}\text { Chromis atrilobata } \\
\text { Gill } 1862\end{array}$ & & 1 & 1 & & & & & $\mathrm{PC}$ & $\mathrm{J}$ & Pla & $\mathrm{S}$ \\
\hline & & $\begin{array}{l}\text { Microspathodon bairdii } \\
\text { (Gill 1862) }\end{array}$ & 1 & 1 & 1 & 1 & 1 & & & Co & $\mathrm{J}$ & Omn & S \\
\hline & & $\begin{array}{l}\text { Microspathodon dorsalis } \\
\text { (Gill 1862) }\end{array}$ & 1 & & 1 & & & & & $\mathrm{PC}$ & $\mathrm{J}$ & Omn & S \\
\hline & & $\begin{array}{l}\text { Stegastes acapulcoensis } \\
\text { (Fowler 1944) }\end{array}$ & 1 & 1 & 1 & 1 & 1 & & 1 & $\mathrm{Ab}$ & $\mathrm{J}$ & Omn & $\mathrm{S} / \mathrm{G}$ \\
\hline & & $\begin{array}{l}\text { Stegastes flavilatus } \\
\text { (Gill 1862) }\end{array}$ & 1 & 1 & 1 & & 1 & & & Co & $\mathrm{J}$ & Omn & $\mathrm{S} / \mathrm{G}$ \\
\hline
\end{tabular}




\begin{tabular}{|c|c|c|c|c|c|c|c|c|c|c|c|c|c|}
\hline Orden & Familia & Nombre científico & $\mathrm{JU}$ & $\mathrm{CU}$ & SE & SO & SJ & PG & PN & Abu. & Est. & Gre. & Com. \\
\hline & Scorpaenidae & $\begin{array}{l}\text { Scorpaenodes xyris } \\
\text { (Jordan \& Gilbert 1882) }\end{array}$ & & 1 & 1 & & 1 & & & $\mathrm{PC}$ & $\mathrm{J}$ & Omn & $\mathrm{S}$ \\
\hline & Serranidae & $\begin{array}{l}\text { Epinephelus labriformis } \\
\text { (Jenyns 1840) }\end{array}$ & 1 & 1 & 1 & 1 & 1 & & 1 & $\mathrm{MC}$ & $\mathrm{J}$ & Carn & $\mathrm{S}$ \\
\hline & & $\begin{array}{l}\text { Rypticus bicolor } \\
\text { Valenciennes } 1846\end{array}$ & 1 & 1 & & 1 & 1 & & 1 & $\mathrm{MC}$ & $\mathrm{J}$ & Carn & S \\
\hline & & $\begin{array}{l}\text { Rypticus nigripinnis } \\
\text { Gill } 1861\end{array}$ & 1 & 1 & 1 & & & & 1 & Co & $\mathrm{J}$ & Carn & S \\
\hline & & $\begin{array}{l}\text { Serranus psittacinus } \\
\text { Valenciennes } 1846\end{array}$ & 1 & 1 & 1 & 1 & 1 & & 1 & Co & $\mathrm{J}$ & Carn & $\mathrm{S}$ \\
\hline \multirow[t]{6}{*}{ Tetraodontiformes } & Diodontidae & $\begin{array}{l}\text { Diodon holocanthus } \\
\text { Linnaeus } 1758\end{array}$ & 1 & & & 1 & & & 1 & $\mathrm{PC}$ & $\mathrm{J}$ & Carn & S \\
\hline & Tetraodontidae & $\begin{array}{l}\text { Arothron hispidus } \\
\text { (Linnaeus 1758) }\end{array}$ & & 1 & & 1 & 1 & & 1 & $\mathrm{PC}$ & $\mathrm{J}$ & Carn & S \\
\hline & & $\begin{array}{l}\text { Arothron meleagris } \\
\text { (Anonymous 1798) }\end{array}$ & 1 & 1 & 1 & 1 & 1 & & & $\mathrm{Co}$ & $\mathrm{J} / \mathrm{A}$ & Carn & S \\
\hline & & $\begin{array}{l}\text { Canthigaster punctatissima } \\
\text { (Günther 1870) }\end{array}$ & & & 1 & & & & & $\mathrm{Ra}$ & $\mathrm{J}$ & Omn & S \\
\hline & & $\begin{array}{l}\text { Sphoeroides lobatus } \\
\text { (Steindachner 1870) }\end{array}$ & & 1 & & 1 & & 1 & & $\mathrm{PC}$ & A & Carn & S \\
\hline & & $\begin{array}{l}\text { Sphoeroides rosenblatti } \\
\text { Bussing } 1996\end{array}$ & 1 & & & & & 1 & & $\mathrm{Ra}$ & $\mathrm{J}$ & Carn & S \\
\hline Total & & & 39 & 39 & 39 & 36 & 36 & 12 & 27 & & & & \\
\hline
\end{tabular}

contenedor frío para su eutanasia y posterior análisis e identificación. Las identificaciones se realizaron siguiendo las claves y guías de campo de Bussing y López (2005), Fisher et al. (1995), y Robertson y Allen (2015). Se obtuvieron muestras de tejido (i.e., una porción de la aleta pectoral derecha y/o musculo de la región latero-dorsal derecha) para la mayoría de los ejemplares recolectados, las cuales fueron preservadas en etanol $96 \%$. Los ejemplares fueron fijados en formol 10 $\%$ y preservados en etanol $70 \%$. Tanto las muestras de tejido como los especímenes de referencia fueron trasladados e ingresados al acervo de la colección ictiológica del Museo de Zoología de la Universidad de Costa Rica (UCR), donde se encuentran disponibles para consulta; muestras duplicadas son resguardadas en la colección ictiológica del Museum of Natural Science de la Louisiana State University (LSU), Estados Unidos, y de la Universidad Michoacana de San Nicolás de Hidalgo (UMSNH), México. Ejemplares de fácil identificación en campo y/o de aquellas especies con alta representatividad numérica en los muestreos fueron trasladados a pozas de "recuperación" para su posterior reincorporación al medio natural, evitando al máximo el sacrificio innecesario de ejemplares. Las recolectas científicas fueron amparadas por los siguientes permisos de investigación: 07-2013-SINAC, 089-2013-SINAC, R-056-2015-OT-CONAGEBIO, ACG-PI-040-2017 y R-SINAC-ACGPI-004-2019 BIOMAR.

Las listas de especies generadas (Tabla 1 y Material Suplementario) siguen a Fricke, Eschmeyer, y Van der Laan (2021) en cuanto a la clasificación jerárquica (familias y órdenes) respectiva y en el uso y validación de los nombres científicos, así como en la designación del autor y año de descripción. Los órdenes, las familias dentro de cada orden y géneros y especies dentro de cada familia se listan alfabéticamente. Datos de abundancias relativas por especie se presentan de forma categórica, basado en un esquema arbitrario de clasificación: especies abundantes (Ab: más de 100 individuos identificados/registrados), muy comunes 
(MC: entre 50 y 99 individuos), comunes (Co: entre 20 y 49 individuos), poco comunes (PC: entre 5 y 19 individuos) y raras (Ra: menos de 5 individuos); y se refieren al esfuerzo de muestreo total, tomando en cuenta el total aproximado de capturas. El estatus de residencia para cada especie (i.e., residente, transitorio o visitante accidental) se asignó siguiendo los criterios de Castellanos-Galindo et al. (2014) y Gibson (1982); los estadios de desarrollo (i.e., juvenil o adulto) se asignaron basados en la distribución de tallas observada en términos generales y las tallas máximas y de madurez reportadas por Robertson y Allen (2015); el gremio trófico (i.e., detritívoro, herbívoro, planctívoro, carnívoro u omnívoro), así como la distribución geográfica generalizada (i.e., restringido o endémico al PTO o de amplia distribución en el Indo-Pacífico) se asignaron según la información provista por Robertson y Allen (2015); y el tipo de hábito (solitario, gregario o críptico) fue asignado tomando en cuenta observaciones realizadas en campo así como datos publicados por González-Murcia et al. (2012), González-Murcia et al. (2016), González-Murcia et al. (2020), y Robertson y Allen (2015).

Análisis comparativo: Datos sobre riqueza de especies ícticas asociadas a ambientes rocosos costeros en diversas zonas geográficas dentro y fuera del PTO (Pacífico Nororiental Templado, i.e., costa este de los Estados Unidos, y Pacífico Suroriental Templado, i.e., costa de Perú-Chile) fueron obtenidos, a efectos comparativos, de la literatura científica. Fueron considerados, en ese sentido, trabajos descriptivos implementando metodologías de captura e inventariado similares o comparables, en la medida de lo posible, a las del presente estudio y tomando como punto de referencia la publicación y lista de referencias de Castellanos-Galindo et al. (2014). La base general para este análisis comparativo está compuesta por datos conjuntos obtenidos a partir de las siguientes ubicaciones y referencias bibliográficas: Norte de California (Moring, 1986; Polivka \& Chotkowski, 1998), Centro de California (Yoshiyama, 1981) y Centro-Sur de California (Boyle \& Horn, 2006), Estados Unidos; Centro-Suroeste de Baja California Sur (Ruiz-Campos et al., 2010), Suroeste de Baja California Sur (Barjau-González et al., 2017; Barjau-González et al., 2019), Norte del Golfo de California (Thomson \& Lehner, 1976) y Michoacán (Torres-Hernández et al., 2016), México; El Pital (González-Murcia et al., 2012) y Los Cóbanos (González-Murcia et al., 2016), El Salvador; ACG (este estudio), Cabo Blanco y Dominical (datos sin publicar), Playas del Coco y Tamarindo (Weaver, 1970), Costa Rica; Isla Gorgona (Castellanos-Galindo et al., 2014) e Isla Palma, Colombia (Castellanos-Galindo et al., 2005); y Norte de Chile (Berrios \& Vargas, 2000), Centro de Chile (Muñoz \& Ojeda, 1997; Stepien, 1990) y Centro-Sur de Chile (Quijada \& Caceres, 2000); totalizando trece localidades dentro de los límites del PTO, cuatro en la costa este de los Estados Unidos y cuatro en la costa de Chile.

Dada la dificultad para estandarizar los datos de abundancia entre los diferentes estudios revisados considerando aspectos propios del esfuerzo de muestreo, tales como el área y volumen de las pozas de marea, sus características físicas (e.g., tipo de sustrato, temperatura, cercanía a la línea de bajamar), área total de muestreo, tiempo total de recolecta, número de recolectores, entre otros, y por tanto de hacer estos datos absolutos o relativos comparables (cuando disponibles), se trabajó solo con datos de presencia/ausencia. Con esta información, se realizó un análisis de similitud, utilizando el índice de Bray-Curtis como medida de diferenciación, por medio del programa de acceso libre PAST (Hammer, Harper, \& Ryan, 2001). Este análisis se realizó a efectos de determinar posibles patrones de agrupamiento y similitud basados en la composición de especies (riqueza) a diferentes escalas de organización local y regional; para esto se siguió además la división biogeográfica propuesta por Ekman (1953), adecuada y revisada por Robertson \& Cramer (2009), donde se consideran, a efectos prácticos, las siguientes subdivisiones/regiones o provincias biogeográficas: Cortez (incluye la 
porción sur de Baja California Sur, el Golfo de California y parte de la costa de Sinaloa, hasta Mazatlán, México) y Mexicana (desde el sur de Mazatlán hasta el oeste de Oaxaca, México), Panámica (desde Chiapas, México hasta el norte de Perú) (dentro del PTO) y Californiana al norte y Peruana-Chilena al sur (fuera del PTO). Basados además en estos datos y la lista general de especies se discuten algunos aspectos e implicaciones biogeográficas. La metodología implementada en este estudio es, en términos generales, comparable a la detallada por Castellanos-Galindo et al. (2014), con algunas adiciones (en cuanto a localidades/número de estudios) y modificaciones puntuales menores (taxonómicas/nomenclaturales, referencias utilizadas, entre otras).

\section{RESULTADOS}

Inventariado (escala local): Se registraron un total de 67 especies de peces óseos asociadas al intermareal rocoso en el ACG; tales especies se encuentran distribuidas en un total de 47 géneros, 24 familias y 11 órdenes. Las familias con mayor riqueza específica fueron: Gobiidae (10 especies), Pomacentridae (8), Gobiesocidae y Labridae, Labrisomidae, Tetraodontidae (5); los órdenes con mayor riqueza específica fueron: Perciformes (23), Gobiiformes (10) y Blenniformes (9). La lista de especies resultante y datos asociados, i.e., presencia/ausencia en los diferentes puntos de muestreo dentro de los límites del ACG, abundancias relativas categóricas y aspectos ecológicos (residencia, gremio trófico, estructura de tallas, tipo de agregación y distribución geográfica generalizada, entre otros), se presentan en la Tabla 1. Algunas especies representativas, de distribución restringida a escala regional y/o de abundancias relativas importantes a nivel local, se ilustran en la Fig. 2.

Dentro del ACG los puntos en Playa La Isla, Bahía Cuajiniquil y Bahía Santa Elena mostraron la mayor diversidad de especies (con 36-39 especies, i.e., 53.7-58.2\% de la diversidad total registrada), mientras que los puntos en Bahía Potrero Grande y Playa
Naranjo mostraron los valores más bajos de diversidad (con 12-27 especies, 17.9-40.3 $\%)$. Del total de especies registrado, 16 (23.9 $\%)$ fueron clasificadas como residentes, 30 (44.8\%) como transitorias y 21 (31.3\%) como visitantes accidentales. Treinta y ocho especies (55.9\% del total) estuvieron representadas solo por individuos juveniles, mientras que 28 (41.2 $\%$ ) especies estuvieron representadas tanto por individuos juveniles como adultos: Sphoeroides lobatus (Steindachner 1870) estuvo representada solo por unos pocos individuos adultos en todo el muestreo. Cuarenta y siete especies (70.1\% del total) presentan hábitos carnívoros, alimentándose principalmente de invertebrados pequeños y en menor medida de otros peces, 13 (19.4\%) especies son omnívoras, 3 (4.5\%) son detritívoras/herbívoras, 3 (4.5\%) planctívoras y una $(1.5 \%)$ herbívora. Treinta y dos especies $(47.8 \%$ del total) presentaron hábitos solitarios, $15(22.4 \%)$ solitarios/crípticos, 9 (13.4\%) solitarios/gregarios/crípticos, 8 (11.9 \%) solitarios/gregarios y 3 (4.5\%) gregarios. A excepción de Echidna nebulosa (Ahl 1789) (de distribución amplia en el océano IndoPacífico), todas las especies registradas en el ACG son endémicas del PTO sensu Robertson y Allen (2015).

Producto del levantamiento regional se listaron un total de 246 especies de peces óseos asociadas al intermareal rocoso del Pacífico americano; tales especies se encuentran distribuidas en 144 géneros, 61 familias y 19 órdenes (Material Suplementario); Labrisomidae (23 especies), Gobiidae (20), Gobiesocidae (17), Cottidae (14), Muraenidae y Pomacentridae (12) fueron las familias más diversas; los órdenes Perciformes (82), Blenniiformes (49), Gobiiformes (23), Gobiesociformes (17) y Anguilliformes (13) fueron, por su parte, los más ricos en especies. En lo que respecta al PTO se registraron 194 especies distribuidas en 116 géneros, 49 familias y 19 órdenes; Gobiidae, Labrisomidae (19 especies), Gobiesocidae (13), Muraenidae (12), Pomacentridae (12) fueron las familias mejor representadas; Perciformes (55), Blenniformes (37), Gobiiformes 

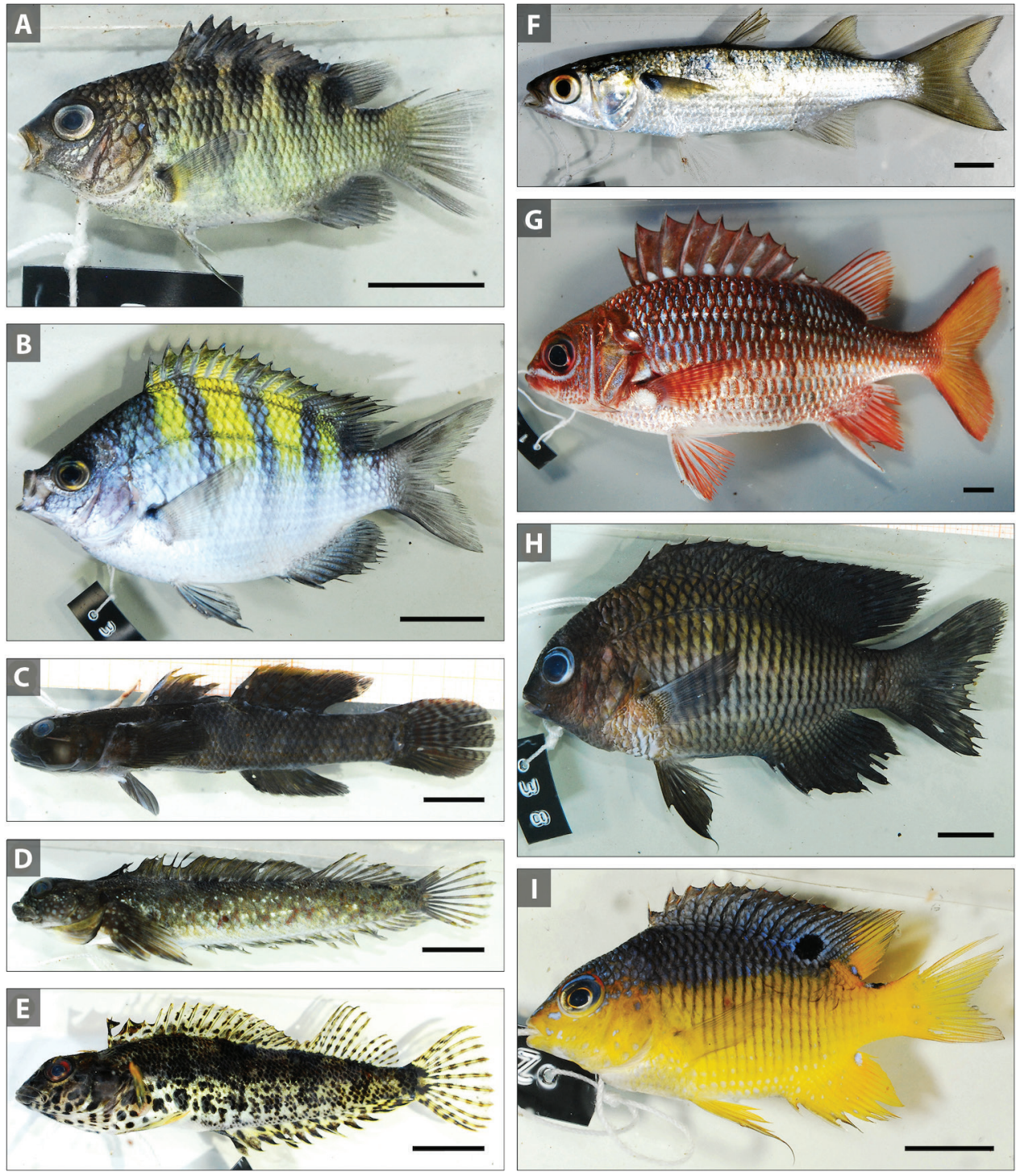

Fig. 2. Especies de peces representativas (por sus abundancias relativas) del intermareal rocoso del ACG, Pacífico Norte de Costa Rica; en orden alfabético: A) Abudefduf concolor (Gill 1862), B) Abudefduf troschelii (Gill 1862), C) Bathygobius ramosus Ginsburg 1947, D) Dialommus macrocephalus (Günther 1861), E) Malacoctenus sudensis Springer 1959, F) Mugil setosus Gilbert 1892, G) Sargocentron suborbitale (Gill 1863), H) Stegastes acapulcoensis (Fowler 1944) y I) Stegastes flavilatus (Gill 1862). Barra de escala $=2 \mathrm{~cm}$.

Fig. 2. Some representative fish species (given their relative abundances) of the rocky intertidal zone of the ACG, North Pacific coast of Costa Rica; in alphabetic order: A) Abudefduf concolor (Gill 1862), B) Abudefduf troschelii (Gill 1862), C) Bathygobius ramosus Ginsburg 1947, D) Dialommus macrocephalus (Günther 1861), E) Malacoctenus sudensis Springer 1959, F) Mugil setosus Gilbert 1892, G) Sargocentron suborbitale (Gill 1863), H) Stegastes acapulcoensis (Fowler 1944) and I) Stegastes flavilatus (Gill 1862). Scale bar $=2 \mathrm{~cm}$. 


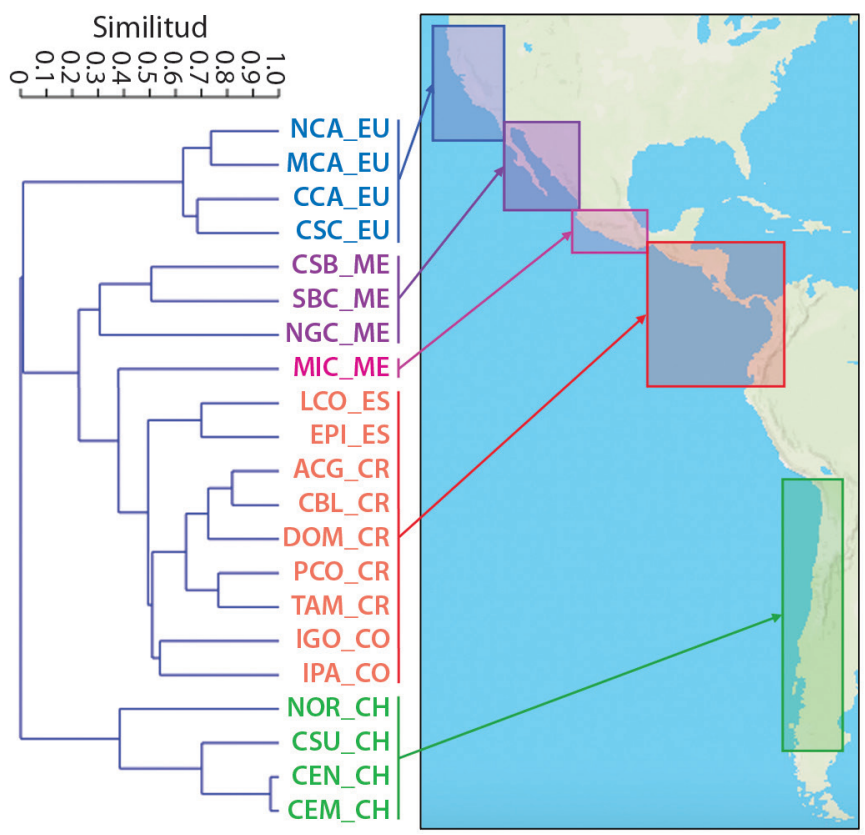

Fig. 3. Resultados del análisis de similitud, basado en datos de presencia/ausencia de especies ícticas asociadas a la zona del intermareal rocoso (ver Material Suplementario), para un total de 21 localidades a lo largo del PTO. Norte de California (NCA_EU y MCA_EU), Centro de California (CCA_EU) y Centro-Sur de California (CSC_EU), Estados Unidos (EU); Centro-Suroeste de Baja California Sur (CSB_ME), Suroeste de Baja California Sur (SBC_ME), Norte del Golfo de California (NGC_ME) y Michoacán (MIC_ME), México (ME); El Pital (EPI_ES) y Los Cóbanos (LCO_ES), El Salvador (ES); Área de Conservación Guanacaste (ACG_CR), Cabo Blanco (CBL_CR), Dominical (DOM_CR), Playas del Coco (PCO_CR), Tamarindo (TAM_CR), Costa Rica (CR); Isla Gorgona (IGO_CO) e Isla Palma (IPA_CO), Colombia (CO); y Norte de Chile (NOR_CH), Centro de Chile (CEN_CH y CEM_CH) y Centro-Sur de Chile (CSU_CH). Los polígonos y colores indican un aproximado del área definida por las provincias ictiográficas Californiana (azul), Cortes (morado), Mexicana (rosado), Panámica (rojo) y Peruana-Chilena (verde).

Fig. 3. Results of the analysis of similarity, based on data of presence/absence of fish species associated to the rocky intertidal zone in a total of 21 localities along the ETP (see Supplementary Material). The polygons and colors indicate an approximate area defined by the ichthyographic provinces Californian (blue), Cortes (purple), Mexicana (pink), Panamica (red) and Peruana-Chilena (green).

(22), Anguilliformes y Gobiesociformes (13) fueron, por su parte, los órdenes más diversos.

\section{Análisis comparativo (escala regional):}

Producto del análisis regional, se obtuvieron tres grupos (conjuntos de localidades) altamente consistentes presentando una composición específica (diversidad) similar y correspondientes, además, con las grandes subdivisiones ictiogeográficas definidas a priori para la costa del Pacífico americano: Pacífico Nororiental Templado (i.e., provincia Californiana), PTO y Pacífico Suroriental Templado (i.e., provincia Peruana-Chilena) (Fig. 3). Dentro del PTO se identificaron además tres subgrupos altamente consistentes y correspondientes con las provincias ictiogeográficas definidas a priori: Cortez (incluyendo las localidades en la costa Sur de Baja California Sur y en el Golfo de California), Mexicana (incluyendo la localidad en Michoacán, en la costa centro-sur Mexicana) y Panámica (incluyendo las localidades en las costas centroamericana y colombiana) (Fig. 3). A lo interno de la provincia Panámica se observó además un agrupamiento latitudinal donde las localidades ubicadas al norte (El Salvador), centro-sur (Costa Rica) y sur (Colombia) presentaron entre sí una mayor similitud en términos de la diversidad (riqueza) ictiológica registrada (Fig. 3). 
El ACG presentó, en términos comparativos, la mayor riqueza específica a nivel regional (con un $27.2 \%$ del total de las especies listadas para el Pacífico americano y un $34.5 \%$ del total de las especies listadas para el PTO), seguida por la localidad de Cabo Blanco, Costa Rica, con 60 especies listadas (24.4\% y 30.9 $\%$ respectivamente), la localidad al CentroSuroeste de Baja California Sur, México, con 58 especies listadas (23.6 \% y $29.9 \%$ ) y las localidades en Michoacán, México, Los Cóbanos, El Salvador e Isla Gorgona, Colombia, con 51 especies listadas $(20.7 \%$ y $26.3 \%)$. El PTO presentó, a su vez, la mayor diversidad de especies a nivel de la costa americana, con una media de $\sim 57$ especies por localidad $(23.3 \%$ del total de la diversidad registrada), disminuyendo tanto hacia el norte como hacia el sur; para la costa este de los Estados Unidos se registraron en promedio $\sim 19$ especies por localidad (7.7 \%) y para la costa de Chile se registraron en promedio $\sim 12$ especies por localidad (14.2 $\%)$. Treinta y cinco de las 49 familias registradas dentro del PTO se restringieron a esta región biogeográfica, cuatro familias (Cottidae, Embiotocidae, Paralichthyidae y Pholidae) fueron compartidas con la porción norte del continente (provincia Californiana), ocho familias (Blenniidae, Clupeidae, Girellidae, Gobiidae, Haemulidae, Labrisomidae, Pomacentridae y Tripterygiidae) fueron compartidas con la porción sur del continente (provincia Peruana-Chilena) y dos familias (Clinidae y Gobiesocidae) presentaron una amplia distribución a lo largo de la costa del continente. La representación de las familias Cebidichthyidae, Hexagrammidae, Liparidae, Scytalinidae y Sebastidae se restringió a la provincia Californiana; por su parte, las familias Aplodactylidae, Bovichtidae, Latridae, Nototheniidae, Ophidiidae y Oplegnathidae solo estuvieron representadas en la provincia Peruana-Chilena.

\section{DISCUSIÓN}

Tomando como base de comparación diversos estudios a escala regional, el ACG alberga una elevada diversidad ictiológica en su intermareal rocoso. Como ejemplo, publicaciones previas dentro del PTO (CastellanosGalindo et al., 2005; Ruiz-Campos et al., 2010, entre otros) reportan, solo entre un $40.3 \%$ y un $86.6 \%$ de la diversidad absoluta registrada en el presente estudio; siendo el ACG, por tanto, la zona (con datos publicados) con la mayor riqueza de especies en el PTO. Esta elevada ictiodiversidad puede ser explicada por una combinación de factores, los cuales incluyen: 1) diferencias en el esfuerzo de muestreo $[\mathrm{El}$ presente estudio incluye/representa un esfuerzo de muestreo de alrededor de 5 años, con un importante número de ubicaciones muestreadas, maximizando la representatividad de microambientes y otras posibles variaciones temporales y estacionales (e.g., temporadas de reproducción, reclutamiento, o fenómenos climáticos, entre otros) a lo largo de este periodo, utilizando además una técnica altamente generalizada permitiendo la detección de especies crípticas, un componente generalmente submuestreado en otros estudios descriptivos]; 2) heterogeneidad ambiental y topografía del ACG [El ACG alberga una alta diversidad de paisajes y ambientes marino-costeros, incluyendo áreas de arrecife rocoso y coralino, manglar y de fondos blandos o arenosos, entre otros (Cortés, 2017), los cuales se encuentran estrechamente interligados e interconectados a la zona intermareal incrementando así su diversidad específica. Además, en diversas porciones del ACG hay intermareal rocoso extenso, con una amplitud de marea considerable (Cortés, 2017), lo cual facilita además el intercambio y predominancia de especies transitorias o accidentales, dada la diversidad de microambientes, tamaño de las pozas de marea y afectación/ grado de exposición al oleaje. Esto contrasta con la costa central de México, por ejemplo, donde la zona intermareal es más estrecha, con una amplitud de mareas menor, y mayor afectación por el oleaje, limitando el acceso de algunas especies dadas sus particularidades de uso de hábitat y exigencias/limitaciones de locomoción y tamaño (Hernández, Álvarez, \& Villalobos, 2010; Torres-Hernández et al., 2016)]; 3) presencia de una rica ictiofauna 
submareal asociada [Además de la elevada heterogeneidad ambiental e interconexión de ambientes, datos publicados (Cortés \& Joyce, 2020) y sin publicar revelan la presencia de más de 400 especies de peces óseos en ambientes marinos del ACG, este número representa cerca de una tercera parte de la ictiodiversidad total registrada en aguas someras $(0-100 \mathrm{~m})$ en el PTO (Robertson \& Allen, 2015); esta elevada diversidad submareal podría verse reflejada en mayor o menor medida en los componentes residente, transitorio y accidental de la zona intermareal]; 4) ubicación geográfica [Estudios previos (e.g., Castellanos-Galindo et al., 2014) han demostrado y descrito cambios en la composición, estructura y diversidad de especies ícticas a lo largo de diversos gradientes latitudinales, con la porción central del PTO siendo la zona más diversa en la costa oeste del continente americano]; 5) ubicación dentro de un área de protección estatal [El ACG comprende un total de 12000 y 43000 ha de área terrestre y marina, respectivamente, así como una amplia y heterogénea línea de costa, las cuales se encuentran formalmente protegidas por ley estatal desde 1986. Dentro de los límites del ACG se impulsan y desarrollan, consecuentemente, diversas actividades, acciones y propuestas de manejo, protección y conservación de sus recursos marinos y terrestres, limitando así posibles afectaciones antropogénicas negativas y repercutiendo de forma directa y positiva sobre los valores de diversidad observados]; entre otros. Lo anterior es consistente, de una u otra forma, con lo discutido en estudios previos desarrollados en otras regiones del globo donde zonas con características similares a las del presente estudio presentan una mayor diversidad ictiológica (y en general) asociada a sus hábitats intermareales (Almada \& Faria, 2004; Arakaki \& Tokeshi, 2006; Gibson \& Yoshiyama, 1999; Griffiths, Davis, \& West, 2006; Horn \& Gibson, 1988; Macieira \& Joyeux, 2011).

De la misma forma, las diferencias en los valores de diversidad absoluta entre los diferentes puntos muestreados a lo largo de la línea de costa del ACG podrían deberse a variaciones (a pequeña escala) en las características y condiciones ambientales y topográficas ya mencionadas. Como ejemplo, los puntos en Playa Potrero Grande y Playa Naranjo presentaron los valores más bajos de diversidad absoluta, lo cual podría estar asociado a una zona intermareal rocosa estrecha o limitada en área (y consecuentemente con una baja estructura topográfica), cercana o influenciada además por un área estuarina (con menor diversidad relativa de especies submareales); en contraposición a los altos valores de diversidad observados en puntos tales como Playa La Isla, Bahía Cuajiniquil y Bahía Santa Elena, los cuales presentan una área de intermareal rocoso comparativamente más extensa y heterogénea, estrechamente asociada, además, a zonas de arrecife rocoso/coralino (con mayor diversidad relativa de especies submareales). El mismo patrón encontrado en el presente estudio fue observado, descrito y discutido por Castellanos-Galindo et al. (2005), Castellanos-Galindo et al. (2014) y Weaver (1970), al comparar (a una escala geográficamente limitada) los elencos ictiológicos asociados a zonas del intermareal rocoso en porciones de las costas de Costa Rica (Playas del Coco y Tamarindo) y Colombia (Isla Gorgona y Bahía Málaga). Tales autores resaltan, de forma diferencial, la importancia o aporte relativo de los componentes residente, transitorio y accidental, definidos en gran medida por el tipo de hábitats submareales asociado a las zonas intermareales (arrecifal vs. estuarino, principalmente; considerando además su diversidad intrínseca según mencionado anteriormente) y por el efecto de otras variables como la lejanía a la costa continental (para el caso de puntual de Isla Gorgona), el tipo y la composición del sustrato en la zona intermareal, tamaño y volumen de las pozas de marea, longitud de la zona intermareal (medida entre la pleamar y bajamar) y la presencia y disponibilidad de macroalgas y/o otros recursos alimenticios, entre otros, esto en concordancia además con lo discutido por autores previos en otras regiones del PTO y del globo (e.g., Castellanos-Galindo \& Giraldo, 2008; Davis, 2000; González-Murcia et al., 2020; Griffiths et al., 2006; Macieira \& Joyeux, 2011). 
En lo que respecta a la composición de especies según su estatus de residencia, nuestros resultados concuerdan con lo reportado y discutido por Thomson y Lehner (1976), en el Golfo de California, y por CastellanosGalindo et al. (2005) y Castellanos-Galindo et al. (2014), en la costa colombiana, donde el conjunto de especies transitorias es dominante sobre el conjunto de especies residentes. Este patrón corresponde a lo esperado, según discutido por Castellanos-Galindo, Krumme y Willis (2010) y Castellanos-Galindo et al. (2014) y lo mencionado con anterioridad, dada la presencia, tanto en el ACG como en algunas de las áreas cubiertas por tales estudios, de zonas submareales adyacentes altamente diversas (i.e., arrecifes rocosos/coralinos) las cuales propician un flujo bidireccional de especies y organismos entre ambos ambientes; contrario a lo que se observa en zonas intermareales con ambientes submareales asociados o adyacentes mucho menos diversos, en donde las especies residentes tienden a ser dominantes en la zona intermareal (dado un flujo de especies y organismos reducido o limitado con la zona submareal). El tamaño de las pozas, la cercanía a la línea costa y la amplitud de las mareas son otras variables que podrían influir en los resultados observados; tal y como discutido por otros autores (González-Murcia et al., 2012; González-Murcia et al., 2016). En ese sentido, zonas con pozas de marea amplias, cercanas a la línea de costa y con un intercambio más directo y constante con las zonas submareales aledañas presentarían mayor riqueza de especies, con el componente transitorio u ocasional mejor representado en términos relativos.

La dominancia de especies, en términos relativos, dado el número total de especies registradas, representadas solo por ejemplares juveniles en el ACG refuerza la idea de la importancia de su zona intermareal en diversos procesos reproductivos y como zona de crianza para especies asociadas a ambientes arrecifales; un patrón recurrente en el PTO (CastellanosGalindo et al., 2005; Castellanos-Galindo et al., 2014; González-Murcia et al., 2012; González-Murcia et al., 2016). Por otra parte, un porcentaje importante de la diversidad total registrada estuvo representada por ejemplares en estadios juvenil y adulto, lo cual estaría asociado en gran parte al componente residente y reflejaría, además, la importancia de este tipo de ambientes para varias especies transitorias y accidentales en diversos procesos ecológicos a lo largo de las diversas etapas de sus ciclos de vida (Castellanos-Galindo et al., 2005; Castellanos-Galindo et al., 2014; González-Murcia et al., 2012; González-Murcia et al., 2016). Por otra parte, en relación con aspectos asociados al estilo de vida de las especies listadas, la dominancia de especies solitarias es, igualmente, un patrón recurrente en el PTO; nuestros resultados son, en ese sentido, similares a los presentados y discutidos por González-Murcia et al. (2012) y González-Murcia et al. (2016) en dos localidades situadas en la costa de El Salvador y podrían ser explicados por: 1) el número (elevado, ofreciendo una gran variedad de opciones), tamaño y volumen de las pozas de marea (relativamente pequeño, limitando el número de ocupantes por cada poza), así como 2) la opción de evitar interacciones antagónicas (competencia intraespecífica) por el uso de los recursos disponibles (e.g., alimento o refugio, entre otros) ante un exceso de ocupantes por cada poza, y 3) la implementación, en paralelo, de otras estrategias anatómicas y etológicas como la cripticidad (vs. el hábito de agrupamiento), para la no detección (y facilidad de escape) por parte de potenciales depredadores, entre otros.

En lo que respecta a gremios tróficos, se observa, en el ACG, una dominancia, en términos del número total de especies registrado, de organismos carnívoros; este patrón es concordante con los resultados de estudios previos en localidades aledañas (e.g., Castellanos-Galindo \& Giraldo, 2008) donde los invertebrados móviles (principalmente cangrejos, copépodos y poliquetos) representan más de un $65 \%$ del total, en términos de unidades de masa, de las presas consumidas por especies ícticas asociadas a ambientes en zonas intermareales, patrón incluso persistente en otras regiones geográficas (Floeter, Ferreira, Dominici-Arosema, 
\& Zalmon, 2004; Wainwright \& Bellwood, 2002). La dominancia de especies carnívoras en el intermareal rocoso del ACG es consecuente además con la alta diversidad y abundancia de invertebrados móviles asociada a este tipo de ambientes, según reportado y discutido por Cortés (2017) y Vargas-Castillo y Cortés (2019). Asimismo, la relativamente baja diversidad de organismos estrictamente detritívoros/herbívoros [correspondiente con lo reportado en estudios previos a escala regional, e.g., Castellanos-Galindo \& Giraldo (2008)] podría deberse a: 1) la relativamente baja disponibilidad de este recurso alimenticio en la zona de estudio (Cortés, 2017; Weaver, 1970) y 2) la alta plasticidad alimenticia de muchos de los organismos habitando la zona intermareal, accediendo a múltiples recursos de forma diferencial según su disponibilidad espacial y temporal, lo cual se ve reflejado paralelamente en el elevado número de especies omnívoras o generalistas (alrededor de un $20 \%$ de la diversidad total); tema que tratan con más detalle Castellanos-Galindo \& Giraldo (2008), Quijada y Caceres (2000) y Weaver (1970).

La dominancia, en términos de la riqueza específica y abundancias relativas, de especies/ taxones pertenecientes a los órdenes Perciformes, Gobiiformes y Blenniformes y de familias tales como Gobiidae, Gobiesocidae, Labridae, Labrisomidae y Pomacentridae en el intermareal rocoso del ACG es un común denominador a nivel regional [i.e., PTO; sensu Robertson y Allen (2015)]. Similar a lo encontrado en este estudio, Castellanos-Galindo et al. (2012) y González-Murcia et al. (2012), reportaron para la Isla Gorgona, Colombia y El Pital, El Salvador, respectivamente, que las familias Gobiidae, Gobiesocidae, Labrisomidae, Mugilidae y Pomacentridae representaron más de un 80 $\%$ de la diversidad total registrada (incluyendo tanto valores de riqueza de especies como abundancias relativas por especie). Por otro lado, pese a no proveer datos detallados de abundancias relativas, dado el enfoque cualitativo del presente estudio, así como a la relativa disparidad en los esfuerzos de muestreo entre ubicaciones y entre pozas de marea, cabe destacar la dominancia en el ACG de especies tales como Malacoctenus sudensis Springer 1959, Bathygobius ramosus Ginsburg 1947, Tomicodon petersii (Garman 1875), Abudefduf troschelii (Gill 1862) y Mugil setosus Gilbert 1892, lo cual concuerda con lo encontrado por otros autores en localidades cercanas a nivel del PTO, incluyendo parte de las costas de El Salvador, Costa Rica y Colombia (CastellanosGalindo et al., 2005; Castellanos-Galindo et al., 2012; Castellanos-Galindo \& Giraldo, 2008; González-Murcia et al., 2012; González-Murcia et al., 2016; Weaver, 1970).

En términos generales, y desde una perspectiva biogeográfica, los resultados del presente estudio son comparables y corresponden bastante bien con lo descrito y encontrado en trabajos previos (e.g. Briggs \& Bowen, 2012; Ekman, 1953; Robertson \& Cramer, 2009). De la misma forma, el patrón de cambio y similitud de especies recuperado a lo largo del gradiente latitudinal norte-sur examinado en el presente estudio es evidente y comparable a lo encontrado, descrito y discutido por Castellanos-Galindo et al. (2014) en su análisis a escala regional. Por otro lado, diferencias puntuales en la composición taxonómica a escala geográfica, reflejadas en los valores de similitud/disimilitud recuperados entre localidades contiguas a lo largo del PTO (y entre estas y otras porciones de la costa continental), como es el caso de las localidades en la costa Central Mexicana, El Salvador y aquellas situadas en Costa Rica y Colombia, podrían deberse, entre otras posibles razones, a la presencia de procesos o barreras vicariantes limitando el flujo e intercambio de especies evitando así una homogeneización de faunas (siendo esto más evidente en familias tales como Gobiesocidae, Gobiidae y Labrisomidae, entre otros, las cuales presentan varios endemismos locales). Al respecto, diversos estudios a nivel regional, utilizando técnicas moleculares (Palmerín-Serrano et al., 2020; Piñeros et al., 2019) y análisis cualitativos (presencia/ausencia de especies; e.g. Briggs \& Bowen, 2012; Ekman, 1953; Hastings, 2000; Robertson \& Cramer, 2009;), enfocados tanto en especies ícticas asociados a zonas 
intermareales como en aquellas asociados a zonas arrecifales en general, han demostrado repetidamente la importancia de barreras tales como las brechas arenosas de Sinaloa y Centroamericana, los giros oceánicos y patrones de corrientes marinas, incluyendo zonas de afloramientos costeros, entre otros, limitando o afectando la capacidad de dispersión (en estadios larvales y adultos) y colonización de nuevos ambientes de tales organismos, resultando así en una importante estructuración poblacional e incluso procesos de especiación a escala local. En ese mismo sentido, gran parte de las especies residentes en el intermareal rocoso y parte de aquellas transitorias asociadas a arrecifes rocosos/coralinos en el PTO, presentando además hábitos crípticos, muestran, por lo general, capacidades físicas de dispersión bastante limitadas, no solo en estadios tempranos [dada una corta duración del estadio larval y ornamentación reducida limitando así su arrastre/traslado por corrientes marinas, entre otras (Aceves-Medina et al., 2004; Beldade, Pedro, y Gonçalves, 2007; Taylor \& Hellberg, 2005; Torres-Hernández et al., 2020)], sino también en estadios adultos [presentando una limitada capacidad de nado y asociaciones restrictas y bastante específicas con determinados tipos de sustratos o inclusive con otros organismos no móviles o de movilidad limitada (Piñeros et al., 2019; Sandoval-Huerta et al., 2019; Taylor \& Hellberg, 2005; entre otros] propiciando o explicando, aunado a lo anterior, el nivel de estructuración, segregación y diferenciación taxonómica observado en el presente estudio. Como ejemplo, el efecto de las barreras arenosas de Sinaloa y Centroamericana (localizadas frente a las costas de Sinaloa, México y en el sur de México-El Salvador, respectivamente) es bastante evidente en el presente estudio separando gráficamente los conjuntos faunísticos del sur de Baja California Sur-Golfo de California, la costa centro-sur Mexicana y la costa centro sur de Centro AméricaColombia, los cuales corresponden, respectivamente, con las definiciones de las provincias biogeográficas Cortez, Mexicana y Panámica, según Ekman (1953) y Hastings (2000). Estos conjuntos faunísticos estarían compuestos o caracterizados, respectivamente, por especies endémicas locales, estrechamente ligadas a la región intermareal y, además, de hábitos crípticos (Robertson \& Allen, 2015) tales como: Aruma histrio (Jordan 1884), Coralliozetus micropes (Beebe \& Tee-Van 1938), Hypsoblennius gentilis (Girard 1854), H. jenkinsi (Jordan \& Evermann 1896) (Cortez); Arcos erythrops (Jordan \& Gilbert 1882), Axoclinus storeyae (Brock 1940), Labrisomus xanti Gill 1860, Malacoctenus zonifer (Jordan \& Gilbert 1882) (Cortez + Mexicana); Arcos decoris Briggs 1969, A. rhodospilus (Günther 1864), Gobiesox daedaleus Briggs 1951, Malacoctenus costaricanus Springer 1959 y M. sudensis Springer 1959 (Panámica), entre otras.

Finalmente, la diversidad total registrada para el intermareal rocoso de la costa oeste americana, aumenta, con los resultados recopilados en el presente estudio y tomando como base de comparación los datos publicados por Castellanos-Galindo et al. (2012), en un $14.9 \%$, pasando de 214 especies previamente listadas a un total de 246 en la presente contribución. Lo anterior, sumado a lo discutido a lo largo del texto, en concordancia además con lo expuesto por autores previos [e.g., Almada \& Faria (2004); Barjau-González et al. (2017); Barjau-González et al. (2019); CastellanosGalindo et al. (2004); Castellanos-Galindo et al. (2014); Castellanos-Galindo et al. (2020); González-Murcia et al. (2012); GonzálezMurcia et al. (2016); González-Murcia et al. (2020); Ruiz-Campos et al. (2010); Thomson \& Lehner (1976); Weaver (1970); entre otros] refuerza la necesidad de realizar más estudios básicos y aplicados, a escala local y regional, con el objetivo principal de ayudar a mejorar nuestro nivel de comprensión y conocimiento sobre diversos aspectos clave de la dinámicafuncionamiento de este tipo de ambientes, de los diversos procesos físico-químico-biológicos que en ellos ocurren, de su riqueza biológica intrínseca y asociada, de su importancia dentro del paisaje marino-costero como un continuo y de las posibles afectaciones que podrían sufrir por el impacto de diversas actividades de 
origen antrópico. En ese sentido, queda además evidente la presencia de vacíos de información en algunas zonas del PTO, como lo son las costas de Guatemala, Nicaragua, Ecuador y Perú, entre otros, así como de algunas islas continentales y oceánicas, cuyo análisis, en mayor o menor detalle, contribuirá al cumplimiento del objetivo planteado y discutido a lo largo de esta contribución.

Declaración de ética: los autores declaran que todos están de acuerdo con esta publicación y que han hecho aportes que justifican su autoría; que no hay conflicto de interés de ningún tipo; y que han cumplido con todos los requisitos y procedimientos éticos y legales pertinentes. Todas las fuentes de financiamiento se detallan plena y claramente en la sección de agradecimientos. El respectivo documento legal firmado se encuentra en los archivos de la revista.

\section{AGRADECIMIENTOS}

Agradecemos el apoyo, durante el trabajo de campo, de las siguientes personas: Caleb McMahan, Carlos Garita, Frank Joyce, Gilbert Ampie, Omar Valencia, Paola Palmerín, William Ludt, Yareli López y Yelba Vega, entre otros. Al personal técnico y administrativo del CIMAR, la Escuela de Biología y el Museo de Zoología, CIBET, UCR, por los apoyos y facilidades brindadas para la ejecución de este trabajo. Al Sistema Nacional de Áreas de Conservación, al Ministerio de Ambiente y Energía y a la Comisión Nacional para la Gestión de la Biodiversidad de Costa Rica por facilitar los permisos de investigación y recolecta científica. Al Área de Conservación Guanacaste y al Guanacaste Dry Forest Conservation Fund, a la Coordinación de la Investigación Científica de la UMSNH y al Consejo Nacional de Ciencia y Tecnología de México por el financiamiento otorgado bajo el marco de los proyectos BioMar-ACG (808B9-508), CIC-UMSNH-2019 y CONACYTCB-2014-240875, respectivamente.

\section{RESUMEN}

Introducción: El intermareal rocoso constituye un paisaje marino costero dominante en el Pacífico Tropical Oriental, albergando comunidades biológicas altamente diversas y funcionalmente complejas. El conocimiento sobre la diversidad y los diversos procesos biológicos que ocurren en tales ambientes es, no obstante, bastante limitado a escala regional.

Objetivo: Cuantificar, describir y comparar (a escala regional) la diversidad ictiológica asociada al intermareal rocoso del Área de Conservación Guanacaste (ACG), Costa Rica, así como evaluar y discutir algunos patrones biogeográficos y ecológicos.

Métodos: Entre 2014 y 2019 se muestrearon un total de 49 pozas de marea en siete ubicaciones dentro del ACG. Se obtuvieron datos sobre diversidad absoluta (presencia/ ausencia de especies), además de algunos datos ecológicos (estatus de residencia, estadio(s) de desarrollo, gremio trófico, hábitos de agrupación y crípticos y distribución generalizada) los cuales se compararon con datos publicados de otros estudios/inventarios llevados a cabo en diversas localidades (20) a lo largo de la costa del Pacífico Oriental. Por medio de un análisis de similitud se evaluaron posibles patrones de agrupamiento entre localidades (según su composición de especies) y, bajo una perspectiva biogeográfica, hipótesis previas de regionalización.

Resultados: Se registraron un total de 67 especies de peces óseos asociadas al intermareal rocoso del ACG, siendo la localidad con datos publicados, más diversa a nivel del Pacífico Oriental. En el ACG los componentes/ categorías transitorio con 30 especies $(44.8 \%)$, juveniles con 38 especies (55.9\%), carnívoros con 47 especies (70.1 $\%$ ), solitarios con 32 especies (47.8\%) y endémico del PTO con 66 especies (98\%), fueron dominantes. A nivel regional se recopilaron datos para un total de 246 especies. Los resultados del análisis de similitud soportaron el esquema de división biogeográfico, definido a priori, a nivel regional, con las localidades ubicadas dentro de las provincias Californiana, Cortez, Mexicana, Panámica y Peruana-Chilena recuperadas como grupos altamente consistentes. Esta estructura es consecuente con el efecto de barreras como las brechas arenosas de Sinaloa y Centroamericana, entre otras, limitando el flujo de especies entre unidades biogeográficas.

Conclusiones: El ACG presenta una elevada diversidad ictiológica asociada a su intermareal rocoso producto de una combinación de factores única a nivel regional. Los resultados de este estudio refuerzan además la necesidad de realizar más estudios básicos y aplicados a diferentes escalas local y regional con el objetivo de ayudar a comprender y conocer mejor diversos aspectos clave de la dinámica y procesos físico-químico-biológicos ocurriendo en las zonas intermareales, de su riqueza biológica asociada, de su importancia dentro del paisaje marino-costero como un continuo y de las posibles afectaciones que estos podrían sufrir debido al impacto de las actividades humanas. 
Palabras clave: biogeografía; Pacífico Tropical Oriental; América Central; ictiofauna; peces; pozas de marea.

\section{REFERENCIAS}

Aceves-Medina, G., Jiménez-Rosenberg, S. P. A., Hinojosa-Medina, A., Funes-Rodríguez, R., SaldiernaMartínez, R. J., \& Smith, P. E. (2004). Fish larvae assemblages in the Gulf of California. Journal of Fish Biology, 65, 832-847.

Almada, V. C., \& Faria, C. (2004). Temporal variation of rocky intertidal resident fish assemblages-patterns and possible mechanisms with a note on sampling protocols. Reviews in Fish Biology and Fisheries, 14(2), 239-250.

Arakaki, S., \& Tokeshi, M. (2006). Short-term dynamics of tidepool fish community: diel and seasonal variation. Environmental Biology of Fishes, 76, 221-235.

Barjau-González, E., Romo-Piñeda, A. K., López-Vivas, J. M., Pérez-Castillo, J., \& Milicua M. B. P. (2017). Variation of the structure of the intertidal fish community of the Pacific coast of Baja California Sur, México. International Journal of Marine Science, 7(48), 455-461.

Barjau-González, E., Armenta-Quintana, J. A., LópezViva, J. M., \& Romero-Vadillo, E. (2019). Taxonomic distinctness of the intertidal fish community on the Pacific coast of Baja California Sur, México. Open Journal of Marine Science, 9, 86-97.

Beldade, R., Pedro, T., \& Gonçalves, E. J. (2007). Pelagic larval duration of 10 temperature cryptobenthic fishes. Journal of fish Biology, 71, 376-382.

Berrios, V. L., \& Vargas, M. E. (2000). Estructura del ensamble de peces intermareales de la costa rocosa del norte de Chile. Revista de Biología Marina y Oceanografia, 35, 73-81.

Briggs, J. C., \& Bowen, B. W. (2012). A realignment of marine biogeographic provinces with particular reference to fish distributions. Journal of Biogeography, $39,12-30$.

Bussing, W. A., \& López, M. I. (2005). Peces de la Isla del Coco y peces arrecifales de la costa Pacífica de América Central Meridional: Guía ilustrada. San José, Costa Rica: Editorial de la Universidad de Costa Rica.

Castellanos-Galindo, G. A., \& Giraldo, A. (2008). Food resource use in a tropical eastern Pacific tidepool fish assemblage. Marine Biology, 153(6), 1023-1035.

Castellanos-Galindo, G. A., Giraldo, A., \& Rubio, E. A. (2005). Community structure of an assemblage of tidepool fishes on a Tropical Eastern Pacific rocky shore, Colombia. Journal of Fish Biology, 67, 392-408.

Castellanos-Galindo, G. A., Krumme, U., \& Willis, T. J. (2010). Tidal influences on fish distributions on tropical eastern Pacific rocky shores (Colombia). Marine Ecology Progress Series, 416, 241-254.

Castellanos-Galindo, G. A., Giraldo, A., \& Zapata, F. A. (2014). Tidepool fish assemblages of Gorgona Island, Colombian Pacific coast: a local and regional comparison. Revista de Biología Tropical, 62, 373-390.

Cortés, J. (2017). Marine biodiversity baseline for Área de Conservación Guanacaste, Costa Rica: published records. ZooKeys, 652, 129-179.

Cortés, J., \& Joyce, F. (2020). BioMar-ACG: A successful partnership to inventory and promulgate marine biodiversity. Biotropica, 52, 1104-1107.

Davis, J. L. D. (2000). Spatial and seasonal patterns of habitat partitioning in a guild of southern California tidepool fishes. Marine Ecology Progress Series, 196, 253-268.

Ekman, S. (1953). Zoogeography of the Sea. London: Sidgwick and Jackson.

Fisher, W., Krupp, F., Schneider, W., Sommer, C., Carpenter, K. E., Niem, V. H. (1995). Vertebrados 1-2. Guía FAO para la identificación de especies para los fines de la pesca-Pacífico Centro- Oriental. Roma: FAO.

Floeter, S. R., Ferreira, C. E. L., Dominici-Arosemena, A., \& Zalmon, I. R. (2004). Latitudinal gradients in Atlantic reef fish communities: trophic structure and spatial use patterns. Journal of Fish Biology, 64, 1680-1699.

Fricke, R., Eschmeyer, W. N., \& Van der Laan, R. (2021). Eschmeyer's Catalog of Fishes: Genera, Species, References (Descargado: 21 de enero de 2021, http:// researcharchive.calacademy.org/research/ichthyology/catalog/fishcatmain.asp).

Gibson, R. N. (1982) Recent studies on the biology of intertidal fishes. Oceanography and Marine Biology Annual Review, 20, 367-414.

Gibson, R. N., \& Yoshiyama, R. M. (1999). Intertidal fish communities. In M. H. Horn, K. L. M. Martin \& M. A. Chotkowski (Eds.), Intertidal Fishes: Life in Two Worlds (pp. 264-296). London, United Kingdom: Academic Press.

González-Murcia, S., Chicas-Batres, F., \& Lovo, M. H. (2016). Community structure and height distribution of intertidal rockpool fish in Los Cóbanos, El Salvador. Pan-American Journal of Aquatic Sciences, 11, 227-242.

González-Murcia, S., Erdmann, S., \& Larios, R. A. (2020). Is this rock pool suitable habitat? Fish diversity in 
intertidal rock pools of El Zonte, El Salvador. Revista Mexicana de Biodiversidad, 91(3), 1-12.

González-Murcia, S., Marín-Martínez, C., \& Ayala-Bocos, A. (2012). Intertidal rockpool icthyofauna of El Pital, La Libertad, El Salvador. Check List, 8(6), 1216-1220.

Griffiths, S. P., Davis, A. R., \& West, R. J. (2006). Role of habitat complexity in structuring temperate rockpool ichthyofaunas. Marine Ecology Progress Series, 313, 227-239.

Hammer, Ø., Harper, D. A., \& Ryan, P. D. (2001). PAST: Paleontological statistics software package for education and data analysis. Palaeontologia electronica, 4(1), 9 .

Hastings, P. A. (2000). Biogeography of the tropical eastern Pacific: distribution and phylogeny of Chaenopsid fishes. Zoological Journal of the Linnean Society, 128(3), 319-335.

Hernández, C., Álvarez, F., \& Villalobos, J. L. (2010). Crustáceos asociados a sustrato duro en la zona intermareal de Montepío, Veracruz, México. Revista Mexicana de Biodiversidad, 81, 141-151.

Horn, M. H., \& Gibson, R. N. (1988) Intertidal fishes. Scientific American, 251, 64-70.

Taylor, M. S., \& Hellberg, M. E. (2005) Marine radiations at small geographic scales: speciation in neotropical reef gobies (Elacatinus). Evolution, 59, 374-385.

Macieira, R. M., \& Joyeux, J. C. (2011). Distribution patterns of tidepool fishes on a tropical flat reef. Fishery Bulletin, 109, 305-315.

Moring, J. R. (1986) Seasonal presence of tidepool fish species in a rocky intertidal zone of northern California, USA. Hydrobiologia, 134, 21-27.

Muñoz, A. A., \& Ojeda, F. P. (1997). Feeding guild structure of a rocky intertidal fish assemblage in Central Chile. Environmental Biology of Fishes, 49, 471-479.

Palmerín-Serrano, P. N., Tavera, J., Espinoza, E., Angulo, A., Martínez-Gómez, J. E., González-Acosta, A. F., \& Domínguez-Domínguez, O. (2020). Evolutionary history of the reef fish Anisotremus interruptus (Perciformes: Haemulidae) throughout the Tropical Eastern Pacific. Journal of Zoological Systematics and Evolutionary Research, 59(1), 148-162.

Piñeros, V. J., Beltrán-López, R. G., Baldwin, C. C., Barraza, E., Espinoza, E., Martínez, J. E., \& DomínguezDomínguez, O. (2019). Diversification of the genus Apogon (Lacepède, 1801) (Apogonidae: Perciformes) in the Tropical Eastern Pacific. Molecular Phylogenetics and Evolution, 132, 232-242.
Polivka, K. M., \& Chotkowski, M. A. (1998). Recolonization of experimentally defaunated tidepools by northeast Pacific intertidal fishes. Copeia, 2, 456-462.

Quijada, P. A., \& Caceres, C. W. (2000). Patrones de abundancia, composición trófica y distribución especial del ensamble de peces intermareales de la zona centro-sur de Chile. Revista Chilena de Historia Natural, 73, 739-747.

Robertson, D. R., \& Allen, G. R. (2015). Shorefishes of the Tropical Eastern Pacific: online information system. Version 2.0. Smithsonian Tropical Research Institute, Balboa, Panamá. Recuperado de https://biogeodb. stri.si.edu/sftep/en/pages

Robertson, D. R., \& Cramer, K. L. (2009). Shore fishes and biogeographic subdivisions of the Tropical Eastern Pacific. Marine Ecology Progress Series, 380, 1-17.

Robertson, D. R., \& Smith-Vaniz, W. F. (2010). Use of clove oil in collecting coral reef fishes for research. Marine Ecology Progress Series, 401, 295-302.

Ruiz-Campos, G., González-Guzmán, S., Ramírez-Valdéz, A., González-Acosta, A. F., Castro-Aguirre, J. L., \& De La Cruz-Agüero, J. (2010). Composition, density and biogeographic affinities of the rocky intertidal fishes on the western coast of the Baja California Peninsula, Mexico. California Cooperative Oceanic Fisheries Investigations, 51, 210-220.

Sandoval-Huerta, E. R., Beltrán-López, R. G., PedrazaMarrón, C. R., Paz-Velásquez, M. A., Angulo, A., Robertson, D. R., Espinoza, E., \& DomínguezDomínguez, O. (2019). The evolutionary history of the goby Elacatinus puncticulatus in the Tropical Eastern Pacific: Effects of habitat discontinuities and local environmental variability. Molecular Phylogenetics and Evolution, 130, 269-285.

Stepien, C. A. (1990). Population structure, diets and biogeographic relationships of a rocky intertidal fish assemblage in central Chile: high levels of herbivory in a temperate system. Bulletin of Marine Science, 47(3), 598-612.

Thomson, D. A., \& Lehner, C. E. (1976). Resilience of a rocky intertidal fish community in a physically unstable environment. Journal of Experimental Marine Biology and Ecology, 22, 1-29.

Torres-Hernández, E., Betancourt-Resendes, I., Díaz-Jaimes, P., Angulo, A., Espinoza, E., \& DomínguezDomínguez, O. (2020). Independent evolutionary lineage of the clingfish Gobiesox adustus (Gobiesocidae) from Isla del Coco, Costa Rica. Revista de Biología Tropical, 68(Suplemento 1), S306-S319.

Torres-Hernández, E., Palacios-Morales, G., RomeroGallardo, S., Salazar-Araujo, P., García-Meraz, A., 
Madrigal-Guridi, X., Del Moral-Flores, L. F., \& Dominguez-Domínguez, O. (2016). Annotated checklist of the coastal ichthyofauna from Michoacán State, Mexico. ZooKeys, 606, 99-126.

Vargas-Castillo, R., \& Cortés, J. (2019). New records of marine decapods and stomatopods in Área de Conservación Guanacaste (ACG): four years of marine biodiversity inventorying. Marine Biodiversity Records, 12(21), 1-8.

Wainwright, P. C., \& Bellwood, D. R. (2002). Ecomorphology of feeding in coral reef fishes. In P. F. Sale (Ed.), Coral Reef Fishes: Dynamics and Diversity in a Complex Ecosystem (pp. 33-55). San Diego, California: Academic Press.
Weaver, P. L. (1970). Species diversity and ecology of tidepool fishes in three Pacific coastal areas of Costa Rica. Revista de Biología Tropical, 17, 165-185.

Yoshiyama, R. M. (1981). Distribution and abundance patterns of rocky intertidal fishes in Central California. Environmental Biology of Fishes, 6, 315-332. 